\title{
Multi-scale analysis of structural variability of Caryophyllaceae saponins by a simplex machine learning approach
}

Soumaya CHEIKH ALI (E-mail: cheikhalisoumaya@gmail.com) ${ }^{\mathrm{a}, \mathrm{d}}$, Muhammad FARMAN (E-mail: farman@qau.edu.pk) ${ }^{\mathrm{b}}$, Asma HAMMAMI-SEMMAR (E-mail: asma.hamami@gmail.com $)^{\mathrm{c}}$, Nabil SEMMAR (E-mail: nabilsemmar5@gmail.com) ${ }^{\mathrm{d}}$,*

${ }^{a}$ University of Carthage, Faculty of Sciences of Bizerte, Tunisia

${ }^{b}$ Quaid-i-Azam University, Department of Chemistry, Islamabad 45320, Pakistan

${ }^{c}$ University of Carthage, Institut National des Sciences Appliquées et Technologies, Tunis, Tunisia

${ }^{d}$ University of Tunis El Manar, Institut Pasteur de Tunis, Laboratory of BioInformatics,

BioMathematics \& BioStatistics, Tunisia

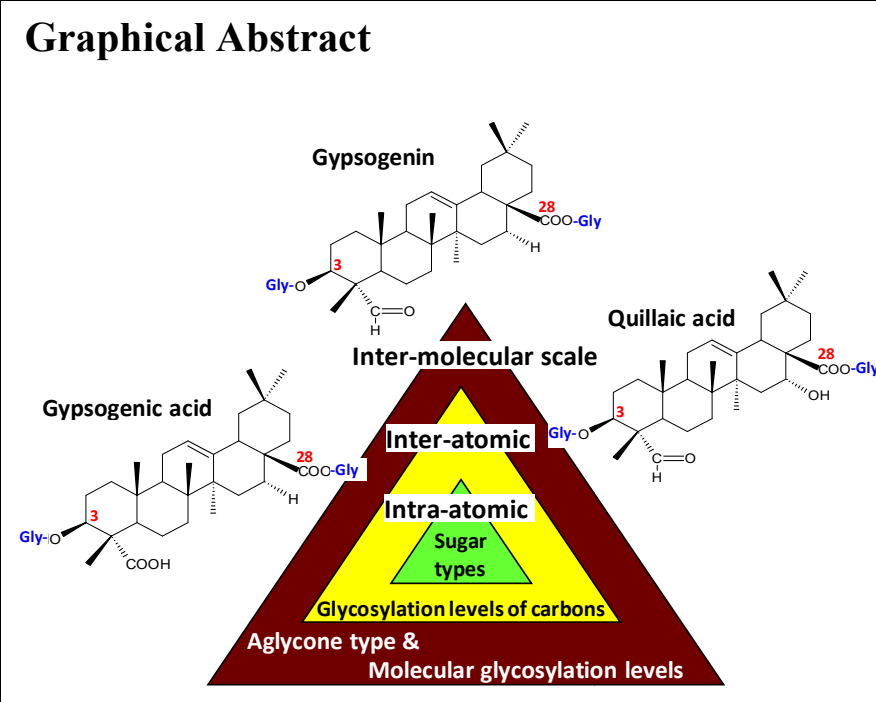

Abstract. A mass conservation law-based chemometric approach was developed to extract smoothed processes governing inter- and intramolecular variability of structural diversity in metabolic pools. The approach consisted of a machine-learning method using simplex rule to calculate a complete set of smoothed barycentric molecules from iterated linear combinations between molecular classes (glycosylation classes). An application to four glycosylation levels $(G L s)$ of Caryophyllaceae saponins highlighted aglycone-dependent variations of glycosylations, especially for gypsogenic acid $(G A)$ which showed high 28-glucosylation levels. Quillaic acid $(Q A)$ and gypsogenin $(G y p)$ showed closer variation ranges of GLs, but differed by relationships between glycosylated carbons toward different sugars. Relative GLs of carbons $\mathrm{C} 3$ and $\mathrm{C} 28$ showed associative (positive), competitive (negative) or independent (unsensitive) trends conditioned by the aglycone type (GA, Gyp) and molecular (total) GLs (the four classes): 28-glucosylation and 28xylosylation showed negative global trends in $G y p$ vs $G L s$-depending trends in $Q A$. Also, relative levels of 3-galactosylation and 3xylosylation varied by unsensitive ways in $G y p$ vs positive trends in $Q A$. These preliminary 
results revealed higher metabolic tensions (competitions) between considered glycosylations in Gyp vs more associative processes in $Q A$. In conclusion, glycosylations of $G A$ and $Q A$ were relatively distant whereas $G y p$ (common precursor) occupied intermediate position.

\section{Introduction}

The Caryophyllaceae plant family was proved to be a wide source of saponins essentially based on three triterpenic skeleton (aglycones or sapogenins) including gypsogenin $(G y p)$, quillaic acid $(Q A)$ and gypsogenic acid $(G A)$ [1].Apart from the sapogenin type, structural variability of Caryophyllaceae saponins showed multi-factorial and multi-scale aspects due to different glycosylation levels (GLs) and glycosylation types essentially occurring at the carbons C3 and C28.

By considering a wide dataset of 205 Caryophyllaceae saponins based onGyp, $Q A$ andGAwith different $G L(2$ to 9), a machine learning approach was applied to extract key information on inter- and intra-molecular regulatory processes governing the observed structural diversityin relation to aglycones (a), glycosylation levelsand types (b, c) and substitution carbons(d) [2]. In silico combinations between saponin structures belonging to different molecular classes $(G L s)$ provided a complete set of simulated theoretical molecules from which significant trends within and between glycosylated carbons were revealed to govern structural variability at inter-molecular scale. This helped to better understand hierarchical and sequential glycosylation orders responsible for diversification of saponins in Caryophyllaceae.

\section{Materials and Methods}

Machine learning approach was applied to the three aglycones separately $(G y p, Q A, G A)$. It consisted in combining structural variabilities of saponins belonging to qmolecular classes (concerning one aglycone)representing qincreasing glycosylation ranges:for $G y p$ and $Q A$, saponins were stratified into $q=4$ classes of glycosylation levels $(G L s)(G L s=1,2,3,4)$ representing saponins with 3-4, 5-6, 7 and 89 substituted sugars, respectively; for GA, $q=3$ classes were considered $(G L s=1,2,3)$ corresponding to saponins with 3, 4, 5 substituted sugars, respectively. Saponins of different $G L$ classes wereinitially characterized by the relative GLs of different sugars substituted at different carbons (C3, C16, C23, C28).Combinations between the qmolecular classes were applied using Scheffé's simplex matrix ( $N$ rows x $q$ columns) which provides a complete set of $N$ mixturesvarying gradually by different weights $w_{j}$ (from $0 / 5$ to $5 / 5$ ) of the qmixed GLclassesj (with $\Sigma w_{j}=1$ )[2].

In output of each combination, a barycentric molecular profile was calculated by averaging the relative levels of glycosylation $(G)$ profiles of the $n$ randomly sampled contributive saponins. The mixture design was iterated 30 times by bootstrap technique then the 30 resulting response matrices (containing $N$ elementary barycentric $G$-profiles) were averaged leading to a final response matrix containing $N$ smoothed barycentric $G$-profiles and representing a deep regulatory machinery of the whole studied structural system.

The smoothed response matrix was used for graphical analysis of regulatory trends between glycosylated carbons.For two given glycosylated carbons, different regulatory trends were highlighted by considering successions of weight ellipses associated to different $G L$ classes [2]. 


\section{Results and Discussion}

Data smoothing by simplexmachine learning approach helped to highlight regulatory processes of glycosylation in Caryophyllaceae saponins at inter-molecular, inter-atomic and intra-atomic scales. Illustrations are provided by xylose $(X y l)$, glucose $(G l c)$ and galactose $(G a l)$ substituted at carbons $\mathrm{C} 3$ and/or C28.

Inter-molecular scale variations 1 (aglycone effect). The three simplex plots associated to the three aglycones-based saponins showed strong differentiation spaces of relationships between the same glycosylated carbons (Figure 1a, b). Illustrations are given for 28- $X y l$ vs 28-Glc (Figure1) and 3- $X y l$ vs 3-Gal (Figure2):

$G A$ was markedly distant from $G y p$ and $Q A$ indicating some specific glycosylation orders (in $G A$ ). This could be linked to the occurrence of two carboxylic groups in $G A(23-$ and 28-COOH) vs only one (28-COOH) in both $Q A$ and Gyp. Specific space of $G A$ was characterized by strong relative 28-Glc levels (0.65-0.75) without competing (comparable) levels from other sugars in both C3 and C28 (Figure1a)[1, 3, 4]. Gyp occupied intermediate position between $G A$ and $Q A$ whereas $Q A$ was the most distant from $G A$ (Figure1a, 2b). Relative locations of different aglycones-based saponins in simulated graphicswere compatible with the metabolism: Gyp is metabolically precursor of both $Q A$ and $G A$ (by 16- and 23-hydroxylation, respectively); this is compatible with intermediate position of Gypbetween $Q A$ and $G A$ (competing for Gyp) [3].

Although Gyp and $Q A$ plots showed spatial neighboring (compared to $G A$ ), they differed by dispersions, inclinations and internal organization of corresponding clouds of points: relationship between 3- $X y l$ and 3-Gal showed significantly higher dispersion in $Q A$ than in Gyp (Figure1b). This aspect indicated wider regulation range of 3-Gal in $Q A$ compared to Gyp[3]. However, for (28-Xyl vs 28-Glc), the two aglycones showed opposite relationship leading to inversely inclined clouds of points: (global positive trend in $Q A$ against negative trend in Gyp) (Figure1a).

Inter-molecular scale variation 2 (molecular glycosylation effect). For a same aglycone, global variation trends between substituted sugars significantly varied with molecular GLs:

In $G y p, 28-G l c$ showed a strong peak under low molecular $G L(G L=1)$ followed by rapid decrease to minimum in higher $G L s(G L=2,3,4)$ (Figure 1b). This highlightedstrong contribution of early 28-Glc in ramification process (monodesmosylation) of $G y p$ leading to preliminary structural diversification of saponins $[3,5,6]$. In $Q A, 28$-Glc seemed to play intermediate modulatory role due to its low relative levels in $G L s=1$ and 4 (figure 1c). 
(a)
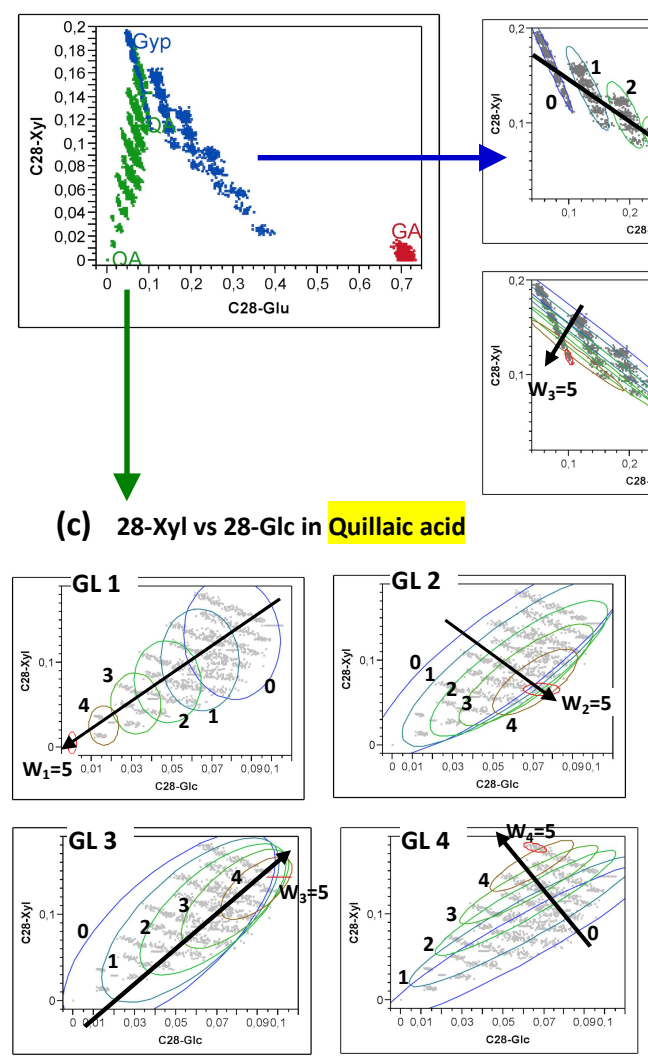

(b) 28-Xyl vs 28-Glc in Gypsogenin
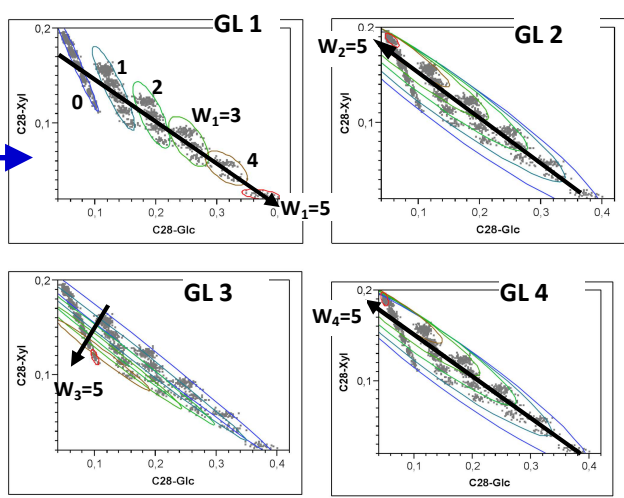

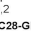

Figure 1. Multidirectional smoothed plots given by simplex machine learning for aglycone-dependent relationships between $\mathrm{C} 28$ xylosylation $(28-X y l)$ and $\mathrm{C} 28$ glucosylation $(28-G l c)$. Three plots corresponding to quillaic acid $(Q A)$, gypsogenin (Gyp) and gypsogenic acid $(G A)$. (b, c) Four plots associated to four glycosylation levels (GLs) in Gyp and $Q A$, respectively.

Figure 2. Multidirectional smoothed plots given by simplex machine learning for aglycone-dependent relationships between $\mathrm{C} 3-$ xylosylation $(3-X y l)$ and $\mathrm{C} 3-$ galoactosylation (28-Gal). (a) Three plots corresponding to quillaic acid $(Q A)$, gypsogenin $(G y p)$ and gypsogenic acid $(G A)$. (b, c) Four plots associated to four glycosylation levels (GLs) in $Q A$ and Gyp, respectively.

However, in Gyp, relative levels of $28-X y l$ showed alternated states with molecular GLs: global minimal regulations in the less glycosylated saponins $(G L=1)$, followed by maximal global regulations in $G L=2$ and 4 via slight decrease in $G L=3$ (Figure 1b). This highlighted key role of $28-X y l$ in 
elongation process for the synthesis of Gyp-based saponins with high GLs. Also, in $Q A, 28-X y l$ showed gradual (step-by-step) increases of relative levels with molecular GLs. This was indicative of key role of xylosylation in elongation process at $\mathrm{C} 28$ in $Q A$ (Figure 1c).

Inter-atomic scale variation. By considering xylosylation between $\mathrm{C} 3$ and $\mathrm{C} 28$ in $Q A, 3$-xylosylation seemed to be initially favored while 28- $X y l$ was at its lowest levels (in $G L=1$ ) (Figures 1c, 2b). This could be indicative of key role of 3-xylosylation in molecular ramification of $Q A$ [3]. For higher $G L$ (2 to 4), relative level of $28-X y l$ showed continuous increase vs alternated variation for $3-X y l$. This highlighted increasing affinity of C28 vs alternated affinity of C3 for xylosylation with GLs in $Q A$.

Intra-atomic scale variation. By considering the inclinations of weight ellipses of different GLs, further regulatory processes were highlighted between $28-X y l$ and $28-G l c$ conditionally to the aglycone type ( $G y p$ vs $Q A$ ) (Figures 1a, 2):

In Gyp, weights' ellipses showed negative inclinations indicating systematic competitions between $X y l$ and $G l c$ for substitution at C28 in all the GLs (Fig. 1b). Such a competition could suggest the implication of different glycosyl-transferases $(G T)$ in the two glycosylations of C28 [3]. Hypothesis on specific $G T$ in Gyp could be also locally indicated by not clearly inclined weight ellipses in the relationship 3-Xyl vs 3-Gal.

However, in $Q A$, weights' ellipses in $(28-X y l$ vs $28-G l c)$ showed positive inclinations indicating some associative process between these two sugars for their 28-substitution despite some global negative trends (for $G L s=2,4$ ). Such associative substitution process could occur under the effect of a same $G T$ having a promiscuity character and leading to sequential glycosylations by different sugars [5, 7-10]. Also, weight ellipses in 3- $X y l$ vs $3-G a l$ showed some positive inclination (except in $G L=1$ ) leading to further indication about intra-atomic associations between considered glycosyls in favor of shared (promiscuity) GT hypothesis.

\section{Conclusions}

Simplex-based machine learning applied to structural variability of Caryophyllaceae saponins highlighted strong differentiation in metabolic glycosylation governed by the aglycone type, molecular $G L$ and substituted carbon. GA was strongly characterized by high levels of 28-Glc followed by Gyp then $Q A$. Effect of GLs was partially associated to key role of 28-Xyl in elongation in both Gyp and $Q A$. At inter-atomic scale, preliminary 28-Glc and 3- Xyl seemed to be responsible for molecular ramification in Gyp and $Q A$, respectively. At intra-atomic scale, 28-Xyl and 28-Glc showed competition in Gyp and association in $Q A$ that could be indicative of different and shared GTs, respectively. Finally, metabolic tensions for glycosylation seemed to decrease from $G A$ to $Q A$ via $G y p$.

\section{References:}

1. Hostettman, K; Marston, A. and Marston, 1995. Saponins. Cambridge University Press, Cambridge, UK, 1995.

2. Sarraj-Laabidi, A.; Messai H.; Hammami-Semmar, A.; Semmar, N. Chemometric analysis of interand intra-molecular diversification factors by a machine learning simplex approach. A review and research on Astragalus saponins. Current Topics in Medicinal Chemistry2017, 17, 2820-2848.

3. Meesapyodsuk, D.; Balsevich, J.; Reed, D.W.; Covello, P.S. Saponin biosynthesis in Saponaria vaccaria. cDNAs encoding $\beta$-amyrin synthase and a triterpene carboxylic acid glucosyltransferase. Plant Physiology2007, 143, 959-969. 
4. Haralampidis, K.; Trojanowska, M. ; Osbourn, A.E. Biosynthesis of triterpenoid saponins in plants. Adv. Biochem. Eng. Biotechnol. 2002, 75, 31-49.

5. Vogt, T.; Jones, P. Glycosyltransferases in plant natural product synthesis: characterization of a supergene family. Trends Plant Sci.2000, 5, 380-386.

6. Li, R.; Reed, DW.; Liu, E.; Bowles, D.J. Phylogenetic analysis of the UDP-glycosyltransferase multigene family of Arabidopsis thaliana. J. Biol. Chem2001, 276, 4338-4343.

7. Augustin, J. M.; Kuzina, V.; Andersen, S. B.; Bak, S. Molecular activities, biosynthesis and evolution of triterpenoid saponins. Phytochemistry 2011,72 (6), 435-457.

8. Hansen, K. S.; Kristensen, C.; Tattersall, D. B.; Jones, P. R.; Olsen, C. E.; Bak, S.; Møller, B. L. The in vitro substrate regiospecificity of recombinant UGT85B1, the cyanohydrin glucosyltransferase from Sorghum bicolor. Phytochemistry2003, 64 (1), 143-151.

9. Kramer, C. M.; Prata, R. T. N.; Willits, M. G.; De Luca, V.; Steffens, J. C.; Graser, G. Cloning and regiospecificity studies of two flavonoid glucosyltransferases from Allium cepa. Phytochemistry2003, 64 (6), 1069-1076.

10. Modolo, L. V.; Blount, J. W.; Achnine, L.; Naoumkina, M. A.; Wang, X.; Dixon, R. A. A functional genomics approach to (iso) flavonoid glycosylation in the model legume Medicago truncatula. Plant Molecular Biology2007, 64 (5), 499-518. 